is complicated by drug-associated side effects in children with T21. However, a good response to treatment with steroid intra-articular joint injections has been observed. Our study has raised a number of questions. Future research to accurately define this disease \& identify best practice with regards to treatment would be invaluable. We advocate that all children with T21 should have annual musculoskeletal examination as part of their health surveillance programme.

Disclosure of Interest: None declared

DOI: 10.1136/annrheumdis-2017-eular.7020

\section{FRI0767-HPR PARTICIPATING IN A MUSCULOSKELETAL RANDOMISED CONTROLLED TRIAL: IDENTIFICATION OF EDUCATION TRAINING NEEDS BY OCCUPATIONAL THERAPISTS AND PHYSIOTHERAPISTS IN THE UK}

J. Adams $^{1,2}$, P. Barratt ${ }^{2}$, S. Bradley ${ }^{3}$, S. Barbosa-Bouças ${ }^{4}$, K. Hislop Lennie ${ }^{5}$, P. White ${ }^{2}$ on behalf of OTTER II Trial working group. ${ }^{1}$ Arthritis Research UK, Centre of Excellence Sport, Ex \& OA; ${ }^{2}$ Faculty of Health Sciences, University of Southampton, Southampton; ${ }^{3}$ Occupational Therapy, Poole General Hospital, Poole: ${ }^{4}$ Department of Psychology, Buckinghamshire New University, High Wycombe; ${ }^{5}$ Health Sciences, University of Southampton, Southampton, United Kingdom

Background: There is an association between clinical teams engaging with research and improvement in the delivery of health services ${ }^{1}$. Randomised controlled trials (RCTs) provide strong evidence to influence practice in musculoskeletal services. For occupational therapists (OTs) and physiotherapists (PTs) implementing RCTs is not yet commonplace. As part of a multi-centred clinical effectiveness and efficacy RCT of splints for thumb base osteoarthritis (OTTER II Trial) we established an education training programme to support clinical therapists deliver the trial across 15 UK hospitals.

Objectives: To evaluate the content of trial training to educate and support OTTER II Trial clinicians in undertaking clinical research roles.

Methods: Two trial training days were run in the North and South of England. Therapists provided details of their clinical trial experience. They were asked to identify one area in which they felt confident and one in which they were not confident in participating in a RCT. These perceived facilitators and barriers were summarised using descriptive statistics and content analysis.

Results: Thirty five clinicians (20 OTs, 15 PTs) attended a training day, 13 (37\%) had no previous experience with clinical trials; $19(54 \%)$ had been involved with at least one previous clinical trial. Clinicians considered they were already confident in; delivering the trial standardised assessment and treatment to patients $n=21(60 \%)$; trusting the OTTER II trial team and their own hospital research departments to support their research role $n=11(31 \%)$; understanding the trial protocol and what needed to be delivered $n=6(17 \%)$ and, being convinced that the trial asked a relevant question $3(9 \%)$. Areas in which clinicians considered they did not have confidence included; the logistics and time management of delivering a RCT in their own hospital $n=18(51 \%)$; the associated trial paperwork to be completed $n=8(23 \%)$; NHS computer access/wifi access for randomisation procedure $n=7(20 \%)$; recruiting participants to time and target $n=6(17 \%)$ and staff capacity to deliver a RCT in the NHS alongside clinical commitments $n=5(14 \%)$. Conclusions: Well documented trial protocols and support from a trusted research team and local hospital research departments were identified as key areas that help clinicians become confident to engage with a national clinical RCT. Clinicians are less confident about managing the practical logistics, staff time and trial paperwork involved in delivering a national RCT. Clinicians identify that they possess core clinical assessment and treatment skills that already equip them to recruit and treat patients as part of a national trial. The logistics of delivering a trial requires ongoing negotiation and support from clinical service managers and the clinical trial teams to ensure clinicians are supported to deliver the RCT to time and target.

References:

[1] Boaz et al 2015 BMJ Open 5:e009415 doi:10.1136/bmjopen-2015-009415 Acknowledgements: The OTTER II Trial is funded by Arthritis Research UK (Grant Ref number 21019).

Disclosure of Interest: None declared

DOI: 10.1136/annrheumdis-2017-eular.1095

\section{FRI0768-HPR MUSCLE WASTING IN OSTEOARTHRITIS MODEL INDUCED BY ANTERIOR CRUCIATE LIGAMENT TRANSECTION}

J.M.D.S. Silva, P.V.G. Alabarse, V.D.O.N. Teixeira, E.C. Freitas, F.H. de Oliveira, R.M.D.S. Chakr, R.M. Xavier. Faculdade de Medicina, UFRGS, Porto Alegre, Brazil

Background: Osteoarthritis $(\mathrm{OA})$ is a chronic joint disease characterized by progressive loss of articular cartilage and abnormal bone formation. Furthermore, there are changes in periarticular muscles, such as loss of muscle mass, strength and function. These features may contribute to functional impairment among patients.

Objectives: This study aimed to investigate the molecular pathways involved in muscle wasting in an animal model of OA induced by anterior cruciate ligament $(\mathrm{ACL})$ transection in rats.
Methods: Female Wistar rats were allocated into two groups: OA (submitted to the ACL transection; $n=9$ ) and SHAM (submitted to surgical procedures without ACL transection; $n=8$ ) [1]. Spontaneous exploratory locomotion, nociception and body weight of animals were evaluated weekly. Twelve weeks after the disease induction, animals were euthanized and the right knee joints were collected for further confirmation of the disease by histopathology, accordingly to OARSI histologic scoring system [2]. Gastrocnemius muscle from the right hind paw were dissected and weighed. Gastrocnemius was used for evaluation of muscle atrophy [3] and protein expression of myostatin, MuRF-1, MyoD and myogenin. Data were compared by Student's $t$ test or ANOVA followed by Tukey's test or ANOVA followed by Mann-Whitney's U-test. The results are expressed as mean values \pm standard deviation (SD) for symmetric variables and as medians with interquartile range for asymmetric variables. Significance was accepted at $P<0.05$.

Results: Histopathology of the right knee joints confirmed the development of the disease in animals from OA group. Gastrocnemius area of animals from OA group had a reduction of about $10 \%$ compared to animals from SHAM group. Protein expression of myostatin was increased in OA group, while myogenin expression was decreased. MuRF-1 and MyoD expression was similar in both OA and SHAM groups. Spontaneous exploratory locomotion, nociception, body weight and weight of gastrocnemius showed no difference between OA and SHAM groups. Conclusions: Gastrocnemius atrophy in $\mathrm{OA}$ induced by $\mathrm{ACL}$ transection involves increased protein expression of myostatin and decreased protein expression of myogenin. In this model, muscle wasting may be linked to myostatin-induced deficits in satellite-cell differentiation due to decreased expression of myogenin.

References:

[1] Elsaid KA, Machan JT, Waller K, Fleming BC, Jay GD. The Impact of Anterior Cruciate Ligament Injury on Lubricin Metabolism and the Effect of Inhibiting Tumor Necrosis Factor alpha on Chondroprotection in an Animal Model. Arthritis and Rheumatism. 2009;60(10):2997-3006.

[2] Gerwin N, Bendele AM, Glasson S, Carlson CS. The OARSI histopathology initiative - recommendations for histological assessments of osteoarthritis in the rat. Osteoarthritis and Cartilage. 2010;18:S24-S34

[3] Filippin LI, Teixeira VN, Viacava PR, Lora PS, Xavier LL, Xavier RM. Temporal development of muscle atrophy in murine model of arthritis is related to disease severity. Journal of Cachexia Sarcopenia and Muscle. 2013:4(3):231-8.

[4] de Oliveira Nunes Teixeira V, Filippin LI, Viacava PR, de Oliveira PG, Xavier RM. Muscle wasting in collagen-induced arthritis and disuse atrophy. Exp Biol Med (Maywood). 2013:238(12):1421-30.

Disclosure of Interest: None declared

DOI: 10.1136/annrheumdis-2017-eular.4856

\section{FRI0769-HPR EVALUATION OF THE EFFECTIVENESS OF DEEP WATER RUNNING FOR THE TREATMENT OF CHRONIC NONSPECIFIC LOW BACK PAIN}

J.S. Arakaki, F.M. Jennings, S.R. Toffolo, J.C. Tamashiro, J. Natour. Rheumatology, Universidade federal de São Paulo- UNIFESP, São Paulo, Brazil

Background: Low back pain (LBP) is one of the most common musculoskeletal conditions and can lead to disability. Aerobic fitness exercises have recently been suggested as important in the management of pain and physical disability of LBP patients, but there are still no studies that prove the best exercise modality for this condition. One proposed modality is the Deep Water Running (DWR) which are aquatic conditioning exercises that simulates normal running on soil.

Objectives: To evaluate the effectiveness of Deep Water Running in the treatment of chronic nonspecific low back pain.

Methods: It was a randomized controlled trial with a duration of 16 weeks with evaluations performed before the intervention and 8 and 16 weeks after the beginning of the training. The population was composed of 60 patients aged over 18 years with diagnosis of chronic nonspecific low back pain. The intervention group performed aerobic conditioning exercise (Deep Water Running) in a heated pool. The sessions had duration of 50 minutes and frequency of 3 times a week for 16 weeks and with $70 \%$ of the maximum heart rate, with $10 \mathrm{bpm}$ less for the difference in the behavior of $\mathrm{HR}$ in aquatic environment. The control group underwent aerobic conditioning by treadmill exercise for 50 minutes, 3 times a week for 16 weeks, and also with $70 \%$ of maximal heart rate. Evaluation instruments: Visual analog pain scale (EVA) in cm; Likert Scale of pain improvement and worsening according to the patient and according to the evaluator; Functional capacity through the Roland-Morris questionnaire and 6-minute walk test; SF-36 for general quality of life; And amount of antiinflammatories used during the study period. Evaluations were performed by an evaluator who was unaware of the patient allocation group.

Results: The two groups were homogeneous regarding most clinical demographic characteristics in the initial evaluation. The two groups showed statistically significant improvement in the variables Roland Morris, EVA for pain and Time up and go, but no statistically significant differences were found between groups. At the Likert scale, the 6-minute walk test and the amount of anti-inflammatories used during the study, no significant differences were found, the two groups remained unchanged. No adverse events were observed in either group during the exercise program

Conclusions: Deep water running aerobic exercise is as effective as treadmill walking in improving pain and functional capacity in patients with chronic nonspecific low back pain. 